\title{
Reseña
}

\section{HISTORIA DEL SERVICIO DE NEUROLOGÍA HOSPITAL DE SAN JOSÉ, BOGOTÁ DC.}

Eduardo Palacios Sánchez MD*

En Colombia, como en Europa y en Norteamérica, la medicina interna y la psiquiatría acompañaron el desarrollo de la neurología. En nuestro medio se inicia en la Universidad Nacional de Colombia donde los primeros profesores en impartir clases sobre el tema fueron Pablo Llinás, Alfonso Uribe Uribe y Edmundo Rico. En los años sesenta del siglo XX, se crean en dos grandes ciudades del país - Bogotá y Medellín - los primeros servicios de neurología liderados en la primera por Ignacio Vergara y Jaime Potes en el Hospital de San Juan de Dios, y por el doctor Andrés Rosselli en el Hospital Militar Central, con el aval de la Universidad del Rosario; de esta institución han egresado una proporción muy significativa de los neurólogos del país. En Medellín el doctor Carlos Santiago Uribe inicia la neurología en el San Vicente de Paul; en los años setenta de ese mismo siglo, se crea el servicio de neurología del Instituto Neurológico de Colombia, dirigido por Eduardo Vallejo con la asesoría de la Pontificia Universidad Javeriana de Bogotá.

En 1977 el doctor Juan Consuegra entonces director médico del Hospital de San José propuso crear el servicio de neurología al doctor Eduardo Palacios, quien en ese momento dirigía el del Hospital Militar y se desempeñaba además como profesor de la Universidad del Rosario.

En el escenario del Hospital de San José eran los neurocirujanos, en cabeza del fundador de esta especialidad doctor Antonio Becerra Lara, quienes tenían a su cargo la cátedra de neurología en la facultad de medicina del Colegio Mayor de Nuestra Señora del Rosario. Habían

* Jefe del Servicio de Neurología del Hospital de San José. Miembro de la Sociedad de Cirugía de Bogotá. Profesor Titular de Neurología, Fundación Universitaria de Ciencias de la Salud. Bogotá DC, Colombia. trabajado como neurólogos aunque por corto tiem Eduardo Vallejo, Ángela Zea de Ángel y Hugo Mejí La colaboración del doctor Becerra y de los tambis neurocirujanos Manuel Palacios y Juan Mendoza Ve fue de gran importancia en la organización del servic de neurología, el cual fue muy pronto aprobado $\mathrm{p}_{\text {i }}$ la Asociación Colombiana de Facultades de Mediciı (ASCOFAME) para el entrenamiento de residente La cátedra quedó a cargo de este servicio y en ella $\mathrm{r}$ solo participaron en forma activa los neurocirujanı mencionados sino también los fisiólogos Carlos $\mathrm{M}_{\mathbf{r}}$ reno y Antonino Barros.

Durante 35 años, que se conmemoran este 2012, han formado de manera ininterrumpida muchos esp cialistas que hoy sobresalen de manera especial en ámbito nacional y en el internacional. Algunos cuy alma mater es la Fundación Universitaria de Cienci: de la Salud y otros de la Universidad del Rosario. S lugar a dudas, todos han realizado importantes contr buciones al servicio y el desarrollo de la especialida Cómo conmemorar este aniversario sin otorgarle cada uno de ellos un espacio, sin duda insuficiente pe con intención genuina de honrar su profesionalismo sus valores humanos y éticos, que continúan dejanc huella indeleble en el ejercicio de la profesión y la ciencia. Varios se han destacado como profesor universitarios y como especialistas en este y en otr servicios de neurología, apoyando la formación de li nuevas generaciones de neurólogos en el país y en exterior.

Agustín Gutiérrez, el primer médico residente en d positar su confianza en este programa de formació ejerce en Villavicencio. Fue secretario de salud, a calde de esta ciudad y representante a la cámara p el departamento del Meta. Miguel Rueda también 1 
ocupado cargos públicos en el país, entre ellos la dirección del INVIMA y el viceministerio de salud; realizó estudios de movimientos anormales en Barcelona y en la actualidad se desempeña en esa rama en la práctica privada. En el ámbito internacional se distinguen como investigadores y en práctica clínica Javier González (neurooncólogo), en el MD Anderson Cancer Center Aurelio Tobón y Raúl Corredor en Miami.

Entre nuestros primeros egresados Gonzalo Melo se dedicó a la neurooncología y en la actualidad trabaja en el Instituto Nacional de Cancerología. Jorge Eslava, ha realizado importantes aportes en el campo de los trastornos del aprendizaje y de la epilepsia. Edgar Osuna, con estudios en alteraciones del sueño, en la actualidad está vinculado con la Fundación Santa Fe de Bogotá. En esta misma institución trabajan Ángela Gómez, Carlos Mayor, especialista en epilepsia y electroencefalografía, y Aristídes Duque.

En otras importantes instituciones del país como la Fundación Cardioinfantil, se desempeñan María Inés Vergara y Jesús Rodríguez quien acaba de terminar su período como presidente de la Asociación Colombiana de Neurología. En la Clínica de la Policía trabaja Patricia Mendoza, hasta hace poco tiempo docente en nuestro servicio por varios años. Martha Ciro fue jefe del Servicio de Neurología, subdirectora médica de esta clínica y en la actualidad es jefe de educación médica de la Universidad del Rosario. En esta misma institución, otro de nuestros egresados y docente, Leonardo Palacios, ejerce como decano de la Facultad de Medicina desde hace una década.

En la Clínica del Country se encuentra Ignacio Salgado, en Saludcoop, Vladimir Gómez, Diana Sierra en Famisanar y Carlos Perdomo en Compensar. Otros de los neurólogos formados en este programa han sobresalido en el campo de la investigación y han contribuido de manera importante a la producción literaria; entre ellos, Manuel Uribe Granja actual director médico de la línea neurológica de Laboratorios Abbott y autor de publicaciones en neurología y neurotoxicología. En la Clínica Colombia hay varios de nuestros egresados, como jefe de neurología Carlos Navas. Colaboran también Martín Vicuña, Mejibe Majul, Gustavo Castro y Joe Muñoz.
Se han especializado en el Hospital de San José y han regresado a sus ciudades de origen, en donde laboran con éxito Judith Sandoval, Carlos Durango (Montería), Ramón Quintero (Valledupar), José Adenis Silva (Ibagué) y Oscar Pradilla jefe del servicio de la clínica Ardila Lulle (Bucaramanga). También fuera de Bogotá se encuentran María Teresa Obando (Armenia), Hernando Rubiano y Carlos Tolosa (Cali), Bernardo Uribe (Manizales) quien además se ha distinguido por sus trabajos en cefaleas, Enrique Urrea (Pereira), Álvaro Romero, Katherine Mantilla, Basilio Wagner y Johanna Valderrama (Medellín), Mauricio Rueda (Bucaramanga) y Magdalena Gómez en Quito (Ecuador).

Han sido jefes del Servicio de Neurología Eduardo Palacios, Paulo Vega, Marcela de la Ossa y Jorge Eslava. Desde hace algo más de quince años ha colaborado en forma activa con la instrucción y la formación de neurólogos Ernesto Ojeda, neurofisiólogo. En la actualidad egresados de nuestro servicio se encuentran vinculados como docentes los profesores Roberto Suárez, jefe de cátedra de Ciencias Básicas de la Fundación Universitaria de Ciencias de la Salud, Leonardo Hernández y Martín Vicuña.

Han sido profesores en San José y se encuentran en el exterior Jimmy Schieman (Atlanta), Ernesto Potes (Virginia), Gustavo Román ganador del premio nacional de medicina y líder en investigación en enfermedad cerebro vascular y demencias en Houston, Estados Unidos.

Contando con los hospitales de San José e Infantil de San José, nuestro grupo de residentes es el más numeroso de los siete programas aprobados para la formación de neurólogos clínicos. Son ellos Adriana Suárez, Iván Gaona, Francy Castro (cuando aparezca esta nota ya habrán terminado su especialización), Rossana Solano, Yuly Osorio, Mauricio Patiño, Angélica Gómez, Melissa Ibarra, Carolina Ramos, Ana Carolina Pulido, Natalia Vega, Laura Barreto, Diana Mejía, Ángel Corredor, Yiroldy Zárate, Jean Paul Vergara, Juan Diego Vargas, Ángela Navas, Sergio Salgado y Andrés Pérez.

El Servicio de Neurología del Hospital de San José se ha distinguido por las labores asistenciales, su interés 
en la academia, la incursión en proyectos investigativos que contribuyen en la producción de conocimiento y por la actualización permanente de su programa educativo y asistencial que incluye entre otros, la adquisición de modernos equipos de neurofisiología que permiten el desarrollo de polisomnografía, potenciales evocados, electromiografía y video telemetría.

Contamos con un grupo de neurólogos jóvenes reconocidos en nuestro medio, Ernesto Ojeda, Martín Vicuña, Leonardo Hernández, Daniel Helmond, Marco Reyes, Javier Triana, Ricardo Gómez, Luis Roa y Natalia Schroeder.
Siempre hemos contado con el apoyo de las directivas del hospital y de la universidad. En medicina interna recordamos en forma especial a Carlos Argáez, José Ignacio Hernández y María Helena Solano; de los directores del hospital a Juan Consuegra, Albertc Villaneda, Jorge Ruiz, Raúl Barrios, Luis Blanco : en especial a Manuel Palacios quien siempre nos $h$ prestado su invaluable apoyo. Importante colaboraciól de los decanos Alfonso Tribín F, de grata recordación Roberto Jaramillo y Sergio Parra. Sin excepción, lo presidentes de la Sociedad de Cirugía, desde nuestr inicio hasta la actualidad han mostrado su mejor dis posición para escucharnos. En tiempos recientes, ha sido de vital importancia para nuestro desarrollo lo doctores Darío Cadena y Jorge Gómez. 